\title{
THE AUSLANDER-REITEN DUALITY VIA MORPHISMS DETERMINED BY OBJECTS
}

\author{
PENGJIE JIAO AND JUE LE*
}

\begin{abstract}
Given an exact category $\mathcal{C}$, we denote by $\mathcal{C}_{l}$ the smallest additive subcategory containing injectives and indecomposable objects which appear as the first term of an almost split conflation. We prove that a deflation is right determined by some object if and only if its intrinsic kernel lies in $\mathcal{C}_{l}$. We give characterizations for $\mathcal{C}$ having Auslander-Reiten duality.
\end{abstract}

\section{INTRODUCTION}

Let $k$ be a commutative artinian ring and $\breve{k}$ be the minimal injective cogenerator. We denote by $D=\operatorname{Hom}_{k}(-, \breve{k})$ the Matlis duality. The categories we consider are $k$-linear Hom-finite Krull-Schmidt and skeletally small.

Recall from [10] that an abelian category $\mathcal{A}$ is said to have Auslander-Reiten duality, if for any objects $X$ and $Y$, there exist natural isomorphisms

$$
\overline{\operatorname{Hom}}_{\mathcal{A}}(Y, \tau X) \simeq D \operatorname{Ext}_{\mathcal{A}}^{1}(X, Y) \simeq \underline{\operatorname{Hom}}_{\mathcal{A}}\left(\tau^{-} Y, X\right) .
$$

Here $\tau$ and $\tau^{-}$are mutually quasi-inverse equivalences between the stable categories of $\mathcal{A}$. More generally, the notion of generalized Auslander-Reiten duality for an exact category $\mathcal{C}$ was introduced in [6, Section 3]. We denote by $\mathcal{C}_{r}$ the smallest additive subcategory of $\mathcal{C}$ containing projectives and indecomposable objects which appear as the third term of an almost split conflation, and by $\mathcal{C}_{l}$ the smallest additive subcategory containing injectives and indecomposable objects which appear as the first term of an almost split conflation. We still have the mutually quasi-inverse equivalences $\tau$ and $\tau^{-}$between stable categories of $\mathcal{C}_{r}$ and $\mathcal{C}_{l}$.

Auslander introduced the notion of morphisms determined by objects in his Philadelphia note [1, which extends Auslander-Reiten theory in some aspects. We study morphisms determined by objects in an exact category $\mathcal{C}$. We prove that a deflation in $\mathcal{C}$ is right determined by some object if and only if its intrinsic kernel lies in $\mathcal{C}_{l}$; see Theorem 5.4. We give characterizations for an object lying in $\mathcal{C}_{r}$ via morphisms determined by objects; see Theorem 6.3.

Following [9] and [5], we introduce the notions of having right stably determined deflations and having left stably determined inflations. We show that the conditions "C has Auslander-Reiten duality", "C has right stably determined deflations" and "C has left stably determined inflations" are equivalent; see Theorem 7.2

The paper is organized as follows. In Sections 2 we recall some basic properties of morphisms determined by objects. In Section 3 we prove that if two objects

Date: August 13, 2018.

2010 Mathematics Subject Classification. 18E10, 16G70.

Key words and phrases. exact category, almost split conflation, Auslander-Reiten duality, morphism determined by object.

* The corresponding author. 
$C$ and $C^{\prime}$ are isomorphic in the projectively stable category, then a deflation is right determined by $C$ if and only if it is right determined by $C^{\prime}$. In Section 4 we prove the following existence theorem: given objects $C \in \mathcal{C}_{r}$ and $Y \in \mathcal{C}$, for certain submodules $H$ of $\operatorname{Hom}_{\mathcal{C}}(C, Y)$, there exists a deflation $\alpha: X \rightarrow Y$ with $H=$

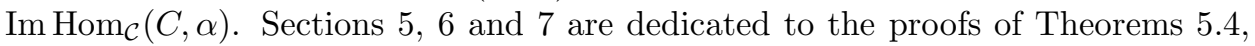
6.3 and 7.2 , respectively.

\section{Morphisms DETERMined By OBJECTS}

Let $\mathcal{C}$ be an additive category. We recall some well-known notions. Here, we use the terminologies in [12, Section 3]. Let $f: X \rightarrow Y$ be a morphism and $C$ be an object. We call $f$ right $C$-determined (or right determined by $C$ ) and call $C$ a right determiner of $f$, if the following condition is satisfied: each morphism $g: T \rightarrow Y$ factors through $f$, provided that for each $h: C \rightarrow T$ the morphism $g \circ h$ factors through $f$. If moreover $C$ is a direct summand of any right determiner of $f$, we call $C$ a minimal right determiner of $f$.

Lemma 2.1. Let $f_{1}: X_{1} \rightarrow Y_{1}$ and $f_{2}: X_{2} \rightarrow Y_{2}$ be two morphisms. Then for an object $C$, the morphism $\left(\begin{array}{cc}f_{1} & 0 \\ 0 & f_{2}\end{array}\right): X_{1} \oplus X_{2} \rightarrow Y_{1} \oplus Y_{2}$ is right $C$-determined if and only if both $f_{1}$ and $f_{2}$ are right $C$-determined.

Proof. For the sufficiency, we assume that $f_{1}$ and $f_{2}$ are right $C$-determined. Let $\left(\begin{array}{l}g_{1} \\ g_{2}\end{array}\right): T \rightarrow Y_{1} \oplus Y_{2}$ be a morphism such that for each $h: C \rightarrow T$, there exists some morphism $\left(\begin{array}{c}s_{1} \\ s_{2}\end{array}\right): C \rightarrow X_{1} \oplus X_{2}$ satisfying $\left(\begin{array}{l}g_{1} \\ g_{2}\end{array}\right) h=\left(\begin{array}{cc}f_{1} & 0 \\ 0 & f_{2}\end{array}\right)\left(\begin{array}{l}s_{1} \\ s_{2}\end{array}\right)$. We obtain $g_{1} \circ h=f_{1} \circ s_{1}$ and $g_{2} \circ h=f_{2} \circ s_{2}$. Since $f_{1}$ and $f_{2}$ are right $C$-determined, there exist some morphisms $t_{1}: T \rightarrow X_{1}$ and $t_{2}: T \rightarrow X_{2}$ such that $g_{1}=f_{1} \circ t_{1}$ and $g_{2}=f_{2} \circ t_{2}$. The morphism $\left(\begin{array}{c}t_{1} \\ t_{2}\end{array}\right): T \rightarrow X_{1} \oplus X_{2}$ satisfies $\left(\begin{array}{c}g_{1} \\ g_{2}\end{array}\right)=\left(\begin{array}{cc}f_{1} & 0 \\ 0 & f_{2}\end{array}\right)\left(\begin{array}{c}t_{1} \\ t_{2}\end{array}\right)$. It follows that $\left(\begin{array}{cc}f_{1} & 0 \\ 0 & f_{2}\end{array}\right)$ is right $C$-determined.

For the necessity, we assume that $\left(\begin{array}{cc}f_{1} & 0 \\ 0 & f_{2}\end{array}\right)$ is right $C$-determined. Let $g: T \rightarrow Y_{1}$ be a morphism such that for each $h: C \rightarrow T$, there exists some morphism $s: C \rightarrow$ $X_{1}$ satisfying $g \circ h=f_{1} \circ s$. The morphisms $\left(\begin{array}{c}g \\ 0\end{array}\right): T \rightarrow Y_{1} \oplus Y_{2}$ and $\left(\begin{array}{l}s \\ 0\end{array}\right): C \rightarrow X_{1} \oplus X_{2}$ satisfy $\left(\begin{array}{l}g \\ 0\end{array}\right) h=\left(\begin{array}{cc}f_{1} & 0 \\ 0 & f_{2}\end{array}\right)\left(\begin{array}{l}s \\ 0\end{array}\right)$. Since $\left(\begin{array}{cc}f_{1} & 0 \\ 0 & f_{2}\end{array}\right)$ is right $C$-determined, there exists some morphism $\left(\begin{array}{l}t_{1} \\ t_{2}\end{array}\right): T \rightarrow X_{1} \oplus X_{2}$ such that $\left(\begin{array}{l}g \\ 0\end{array}\right)=\left(\begin{array}{cc}f_{1} & 0 \\ 0 & f_{2}\end{array}\right)\left(\begin{array}{l}t_{1} \\ t_{2}\end{array}\right)$. We obtain $g=f_{1} \circ t_{1}$. It follows that $f_{1}$ is right $C$-determined. Similarly, the morphism $f_{2}$ is right $C$ determined.

Lemma 2.2. Let $f: X \rightarrow Y$ and $g: Z \rightarrow Y$ be two morphisms. Assume that $f^{\prime}: E \rightarrow Z$ and $g^{\prime}: E \rightarrow X$ form the pullback of $f$ and $g$. If $f$ is right $C$-determined for some object $C$, then $f^{\prime}$ is also right $C$-determined.

Proof. Let $h: T \rightarrow Z$ be a morphism such that for each $u: C \rightarrow T$, there exists some morphism $v: C \rightarrow E$ satisfying $h \circ u=f^{\prime} \circ v$. Then we have $g \circ h \circ u=$ $g \circ f^{\prime} \circ v=f \circ g^{\prime} \circ v$. Since $f$ is right $C$-determined, there exists some morphism $s: T \rightarrow X$ such that $g \circ h=f \circ s$. Since $f^{\prime}$ and $g^{\prime}$ form the pullback of $f$ and $g$, there exists a morphism $t: T \rightarrow E$ such that $h=f^{\prime} \circ t$. It follows that $f^{\prime}$ is right $C$-determined.

Let $f: X \rightarrow Y$ be a morphism. Recall that $f$ is called right almost split if $f$ is not a retraction and each morphism $g: Z \rightarrow Y$ which is not a retraction factors 
through $f$. Dually, we call $f$ left almost split if $f$ is not a section and each morphism $g: X \rightarrow Z$ which is not a section factors through $f$.

Given two objects $X$ and $Y$, we denote by $\operatorname{rad}_{\mathcal{C}}(X, Y)$ the set of morphisms $f: X \rightarrow Y$, that for any object $Z$ and any morphisms $g: Z \rightarrow X$ and $h: Y \rightarrow Z$, the morphism $h \circ f \circ g$ lies in $\operatorname{rad}_{\operatorname{End}}(Z)$. Then $\operatorname{rad}_{\mathcal{C}}$ forms an ideal of $\mathcal{C}$. We observe by [8, Corollary 2.10] that

$$
\operatorname{rad}_{\mathcal{C}}(X, Y)=\left\{f: X \rightarrow Y \mid f \circ g \in \operatorname{rad}_{\operatorname{End}_{\mathcal{C}}}(Y), \text { for each } g: Y \rightarrow X\right\} .
$$

A morphism $g: Z \rightarrow Y$ is said to almost factor through $f: X \rightarrow Y$, if $g$ does not factor through $f$, and for each object $T$ and each morphism $h \in \operatorname{rad}_{\mathcal{C}}(T, Z)$, the morphism $g \circ h$ factors through $f$.

Proposition 2.3. Let $f: X \rightarrow Y$ and $g: Z \rightarrow Y$ be two morphisms, such that $\operatorname{End}_{\mathcal{C}}(Z)$ is a local ring and $g$ almost factors through $f$. Assume that $f^{\prime}: E \rightarrow Z$ is the pullback of $f$ along $g$. Then $f^{\prime}$ is right almost split.

Proof. We observe that $f^{\prime}$ is not a retraction, since $g$ does not factors through $f$. Given an object $T$, assume that $h: T \rightarrow Z$ is not a retraction. We observe by (2.1) that $h \in \operatorname{rad}_{\mathcal{C}}(T, Z)$, since $\operatorname{End}_{\mathcal{C}}(Z)$ is local. Since $g$ almost factors through $f$, there exists some morphism $s: T \rightarrow X$ such that $g \circ h=f \circ s$. By the pullback diagram, there exists a morphism $t: T \rightarrow E$ such that $h=f^{\prime} \circ t$. It follows that $f^{\prime}$ is right almost split.

\section{Deflations Determined By oBJeCts}

Let $k$ be a commutative artinian ring. We denote by $k$-mod the category of finitely generated $k$-modules. Let $\check{k}$ be the minimal injective cogenerator for $k$. Then the Matlis duality $D=\operatorname{Hom}_{k}(-, \breve{k})$ is a self-duality of $k$-mod. From now on, the categories we consider are $k$-linear Hom-finite and Krull-Schmidt.

Let $\mathcal{C}$ be an exact category. Recall that an exact category is an additive category $\mathcal{C}$ together with a collection $\mathcal{E}$ of kernel-cokernel pairs which satisfies the axioms in 1, Appendix A]. Here, a kernel-cokernel pair means a sequence of morphisms $X \stackrel{i}{\rightarrow} E \stackrel{d}{\rightarrow} Y$, which we denote by $(i, d)$, such that $i$ is the kernel of $d$, and $d$ is the cokernel of $i$. Each kernel-cokernel pair $(i, d) \in \mathcal{E}$ is called a conflation, while $i$ is called an inflation and $d$ is called a deflation. Given a conflation $\eta: X \rightarrow E \rightarrow Y$, for each $f: Z \rightarrow Y$ we denote by $\eta$. $f$ the conflation $\operatorname{Ext}_{\mathcal{C}}^{1}(f, X)(\eta)$; for each $g: X \rightarrow Z$ we denote by $g . \eta$ the conflation $\operatorname{Ext}_{\mathcal{C}}^{1}(Y, g)(\eta)$.

Let $\alpha_{1}: X_{1} \rightarrow Y_{1}$ and $\alpha_{2}: X_{2} \rightarrow Y_{2}$ be two morphisms. We mention the fact that $\left(\begin{array}{cc}\alpha_{1} & 0 \\ 0 & \alpha_{2}\end{array}\right): X_{1} \oplus X_{2} \rightarrow Y_{1} \oplus Y_{2}$ is a deflation if and only if both $\alpha_{1}$ and $\alpha_{2}$ are deflations; see [4, Proposition 2.9 and Corollary 2.18].

Recall from [10, Section 2] that a morphism $f: X \rightarrow Y$ is called projectively trivial if for each $Z$, the induced map $\operatorname{Ext}_{\mathcal{C}}^{1}(f, Z): \operatorname{Ext}_{\mathcal{C}}^{1}(Y, Z) \rightarrow \operatorname{Ext}_{\mathcal{C}}^{1}(X, Z)$ is zero. We observe that $f$ is projectively trivial if and only if $f$ factors through each deflation ending at $Y$. Dually, we call $f$ injectively trivial if for each $Z$, the induced map $\operatorname{Ext}_{\mathcal{C}}^{1}(Z, f): \operatorname{Ext}_{\mathcal{C}}^{1}(Z, X) \rightarrow \operatorname{Ext}_{\mathcal{C}}^{1}(Z, Y)$ is zero.

Given two objects $X$ and $Y$, we denote by $\mathcal{P}(X, Y)$ the set of projectively trivial morphisms from $X$ to $Y$. Then $\mathcal{P}$ forms an ideal of $\mathcal{C}$. The projectively stable category $\underline{\mathcal{C}}$ of $\mathcal{C}$ is the factor category $\mathcal{C} / \mathcal{P}$. Given a morphism $f: X \rightarrow Y$, we denote by $\underline{f}$ its image in $\underline{\mathcal{C}}$. We denote by $\underline{\operatorname{Hom}}_{\mathcal{C}}(X, Y)=\operatorname{Hom}_{\mathcal{C}}(X, Y) / \mathcal{P}(X, Y)$ the set of morphisms from $X$ to $Y$ in $\underline{\mathcal{C}}$. 
Dually, we denote by $\mathcal{I}(X, Y)$ the set of injectively trivial morphisms from $X$ to $Y$. The injectively stable category $\overline{\mathcal{C}}$ of $\mathcal{C}$ is the factor category $\mathcal{C} / \mathcal{I}$. Given a morphism $f: X \rightarrow Y$, we denote by $\bar{f}$ its image in $\overline{\mathcal{C}}$. We denote by $\overline{\operatorname{Hom}}_{\mathcal{C}}(X, Y)=$ $\operatorname{Hom}_{\mathcal{C}}(X, Y) / \mathcal{I}(X, Y)$ the set of morphisms from $X$ to $Y$ in $\overline{\mathcal{C}}$.

Lemma 3.1. Let $\alpha: X \rightarrow Y$ be a deflation. Then a morphism $f: T \rightarrow Y$ factors through $\alpha$ in $\mathcal{C}$ if and only if $\underline{f}$ factors through $\underline{\alpha}$ in $\underline{\mathcal{C}}$.

Proof. It is sufficient to show the sufficiency. Assume that $\underline{f}$ factors through $\underline{\alpha}$ in $\underline{\mathcal{C}}$. Then there exists some morphism $g: T \rightarrow X$ in $\mathcal{C}$ such that $\underline{f}=\underline{\alpha} \circ \underline{g}$ in $\underline{\mathcal{C}}$. We have that $f-\alpha \circ g$ is projectively trivial in $\mathcal{C}$. Since $\alpha$ is a deflation, there exists some morphism $h: T \rightarrow X$ such that $f-\alpha \circ g=\alpha \circ h$ in $\mathcal{C}$. It follows that $f=\alpha \circ(g+h)$, factoring through $\alpha$ in $\mathcal{C}$.

Lemma 3.2. Let $C$ be an object. Then a deflation $\alpha: X \rightarrow Y$ is right $C$-determined in $\mathcal{C}$ if and only if $\underline{\alpha}$ is right $C$-determined in $\underline{\mathcal{C}}$.

Proof. For the sufficiency, we assume that $\underline{\alpha}$ is right $C$-determined in $\underline{\mathcal{C}}$. Let $f: T \rightarrow$ $Y$ be a morphism in $\mathcal{C}$ such that for each $g: C \rightarrow T$, the morphism $f \circ g$ factors through $\alpha$ in $\mathcal{C}$. Then $\underline{f} \circ \underline{g}$ factors through $\underline{\alpha}$ in $\underline{\mathcal{C}}$. Since $\underline{\alpha}$ is right $C$-determined in $\underline{\mathcal{C}}$, we have that $f$ factors through $\underline{\alpha}$ in $\underline{\mathcal{C}}$. By Lemma 3.1, the morphism $f$ factors through $\alpha$ in $\mathcal{C}$. It follows that $\alpha$ is right $C$-determined in $\mathcal{C}$.

For the necessity, we assume that $\alpha$ is right $C$-determined in $\mathcal{C}$. Let $f: T \rightarrow Y$ be a morphism in $\mathcal{C}$ such that for each $g: C \rightarrow T$, the morphism $f \circ g$ factors through $\underline{\alpha}$ in $\underline{\mathcal{C}}$. By Lemma 3.1, the morphism $f \circ g$ factors through $\alpha$ in $\overline{\mathcal{C}}$. Since $\alpha$ is right $C$-determined in $\mathcal{C}$, we have that $f$ factors through $\alpha$ in $\mathcal{C}$. Then $f$ factors through $\underline{\alpha}$ in $\underline{\mathcal{C}}$. It follows that $\underline{\alpha}$ is right $C$-determined in $\underline{\mathcal{C}}$.

Proposition 3.3. Let $C$ and $C^{\prime}$ be two objects. Assume that $C \simeq C^{\prime}$ in $\underline{\mathcal{C}}$. Then a deflation $\alpha: X \rightarrow Y$ is right $C$-determined if and only if it is right $C^{\prime}$-determined.

Proof. We observe that $\underline{\alpha}$ is right $C$-determined in $\underline{\mathcal{C}}$ if and only if $\underline{\alpha}$ is right $C^{\prime}$ determined in $\underline{\mathcal{C}}$. Then the result follows by applying Lemma 3.2 twice.

\section{An existence theorem}

Let $\mathcal{C}$ be a Hom-finite Krull-Schmidt exact category. Given an object $C$, we denote by $\Gamma_{C}=\operatorname{End}_{\mathcal{C}}(C)^{\text {op }}$ the opposite algebra of the endomorphism algebra of $C$, and by add $C$ the category of direct summands of finite direct sums of $C$.

Recall from [6, Section 2] two full subcategories $\mathcal{C}_{r}$ and $\mathcal{C}_{l}$ of $\mathcal{C}$ as follows:

$$
\mathcal{C}_{r}=\left\{X \in \mathcal{C} \mid \text { the functor } D \operatorname{Ext}_{\mathcal{C}}^{1}(X,-): \overline{\mathcal{C}} \rightarrow k \text {-mod is representable }\right\}
$$

and

$$
\mathcal{C}_{l}=\left\{X \in \mathcal{C} \mid \text { the functor } D \operatorname{Ext}_{\mathcal{C}}^{1}(-, X): \underline{\mathcal{C}} \rightarrow k \text {-mod is representable }\right\} .
$$

Then we have the mutually quasi-inverse equivalences

$$
\tau: \underline{\mathcal{C}_{r}} \stackrel{\sim}{\longrightarrow} \overline{\mathcal{C}_{l}} \text { and } \tau^{-}: \overline{\mathcal{C}_{l}} \stackrel{\sim}{\longrightarrow} \underline{\mathcal{C}_{r}} .
$$

For each $X \in \mathcal{C}_{r}$, we have a natural isomorphism

$$
\overline{\operatorname{Hom}}_{\mathcal{C}}(-, \tau X) \stackrel{\sim}{\longrightarrow} D \operatorname{Ext}_{\mathcal{C}}^{1}(X,-) ;
$$

for each $X \in \mathcal{C}_{l}$, we have a natural isomorphism

$$
\underline{\operatorname{Hom}}_{\mathcal{C}}\left(\tau^{-} X,-\right) \stackrel{\sim}{\longrightarrow} D \operatorname{Ext}_{\mathcal{C}}^{1}(-, X) .
$$


Recall that a conflation $\eta: X \stackrel{u}{\rightarrow} E \stackrel{v}{\rightarrow} Y$ is almost split if $u$ is left almost split and $v$ is right almost split. Assume that $\eta$ is almost split. We mention that both $X$ and $Y$ are indecomposable. By the following lemma, which is due to [6. Proposition 2.4], we have $X \in \mathcal{C}_{l}$ and $Y \in \mathcal{C}_{r}$. We mention that $Y \simeq \tau^{-} X$ in $\underline{\mathcal{C}}$ and $X \simeq \tau Y$ in $\overline{\mathcal{C}}$; see the proof of [6, Lemma 3.2].

Lemma 4.1. Let $X$ be an indecomposable object.

(1) Assume that $X$ is non-projective. Then $X \in \mathcal{C}_{r}$ if and only if there exists an almost split conflation ending at $X$.

(2) Assume that $X$ is non-injective. Then $X \in \mathcal{C}_{l}$ if and only if there exists an almost split conflation starting at $X$.

The classical case of the following lemma is well known; see [12, Corollary 3.5].

Lemma 4.2. Let $\alpha: X \rightarrow Y$ be a deflation with $\operatorname{Ker} \alpha \in \mathcal{C}_{l}$. Then $\alpha$ is right $\tau^{-}(\operatorname{Ker} \alpha)$-determined.

Proof. Let $f: T \rightarrow Y$ be a morphism such that for each $g: \tau^{-}(\operatorname{Ker} \alpha) \rightarrow T$, the morphism $f \circ g$ factors through $\alpha$. We denote by $\eta$ the conflation Ker $\alpha \rightarrow X \stackrel{\alpha}{\rightarrow} Y$. We obtain that $\eta .(f \circ g)$ splits. Since Ker $\alpha \in \mathcal{C}_{l}$, there exists a natural isomorphism

$$
\phi: \underline{\operatorname{Hom}}_{\mathcal{C}}\left(\tau^{-}(\operatorname{Ker} \alpha),-\right) \longrightarrow D \operatorname{Ext}_{\mathcal{C}}^{1}(-, \operatorname{Ker} \alpha) .
$$

Set $\gamma=\phi_{\tau^{-}(\operatorname{Ker} \alpha)}\left(\underline{\left.\operatorname{Id}_{\tau^{-}(\operatorname{Ker} \alpha)}\right)}\right)$ By the naturality of $\phi$, we have

$$
\phi_{T}(\underline{g})=D \operatorname{Ext}_{\mathcal{C}}^{1}(g, \operatorname{Ker} \alpha)(\gamma)=\gamma \circ \operatorname{Ext}_{\mathcal{C}}^{1}(g, \operatorname{Ker} \alpha) .
$$

It follows that

$$
\phi_{T}(\underline{g})(\eta \cdot f)=\gamma((\eta \cdot f) \cdot g)=\gamma(\eta \cdot(f \circ g))=0 .
$$

We observe that $\phi_{T}(\underline{g})$ runs over all maps in $D \operatorname{Ext}_{\mathcal{C}}^{1}(T, \operatorname{Ker} \alpha)$, when $\underline{g}$ runs over all morphisms in $\underline{\operatorname{Hom}}_{\mathcal{C}}\left(\tau^{-}(\operatorname{Ker} \alpha), T\right)$. It follows that $\eta$.f splits. In other words, the morphism $f$ factors through $\alpha$. Then the result follows.

Given an object $C$ and a morphism $f: X \rightarrow Y$, we denote by $\operatorname{Im} \operatorname{Hom}_{\mathcal{C}}(C, f)$ the image of the induced map $\operatorname{Hom}_{\mathcal{C}}(C, f): \operatorname{Hom}_{\mathcal{C}}(C, X) \rightarrow \operatorname{Hom}_{\mathcal{C}}(C, Y)$. Generalizing 2. Corollary XI.3.4] to an exact category $\mathcal{C}$, we obtain the following existence theorem; compare [2, Theorem XI.3.6] and [12, Theorem 4.4]. Since $\mathcal{C}$ does not have enough projectives, the treatment here is completely different.

Theorem 4.3. Let $C$ and $Y$ be two objects. Assume that $C \in \mathcal{C}_{r}$ and $H$ is a left $\Gamma_{C}$-submodule of $\operatorname{Hom}_{\mathcal{C}}(C, Y)$ satisfying $\mathcal{P}(C, Y) \subseteq H$. Then there exists some deflation $\alpha: X \rightarrow Y$, which is right $C$-determined such that $\operatorname{Ker} \alpha \in \operatorname{add}(\tau C)$ and $H=\operatorname{Im}_{H^{\prime}}(C, \alpha)$.

Proof. By the assumption we have $\tau C \in \mathcal{C}_{l}$ and $\tau^{-} \tau C \simeq C$ in $\underline{\mathcal{C}}$. Then there exists a natural isomorphism

$$
\phi: \underline{\operatorname{Hom}}_{\mathcal{C}}(C,-) \longrightarrow D \operatorname{Ext}_{\mathcal{C}}^{1}(-, \tau C) .
$$

Set $\gamma=\phi_{C}\left(\underline{\operatorname{Id}_{C}}\right)$. By the naturality of $\phi$, for each object $Z$ and each morphism $f: C \rightarrow Z$, we have

$$
\phi_{Z}(\underline{f})=D \operatorname{Ext}_{\mathcal{C}}^{1}(f, \tau C)(\gamma)=\gamma \circ \operatorname{Ext}_{\mathcal{C}}^{1}(f, \tau C) .
$$

Then for each $\mu \in \operatorname{Ext}_{\mathcal{C}}^{1}(Z, \tau C)$, we have

$$
\phi_{Z}(\underline{f})(\mu)=\gamma\left(\operatorname{Ext}_{\mathcal{C}}^{1}(f, \tau C)(\mu)\right)=\gamma(\mu . f) .
$$


We set $\underline{H}=H / \mathcal{P}(C, Y)$ and set

$$
\underline{H}^{\perp}=\left\{\mu \in \operatorname{Ext}_{\mathcal{C}}^{1}(Y, \tau C) \mid \phi_{Y}(\underline{h})(\mu)=0 \text { for each } \underline{h} \in \underline{H}\right\} .
$$

We observe that $\underline{H}^{\perp}$ is a right $\Gamma_{C}$-submodule of $\operatorname{Ext}_{\mathcal{C}}^{1}(Y, \tau C)$. Here, for any $f \in \Gamma_{C}$ and $\mu \in \operatorname{Ext}_{\mathcal{C}}^{1}(Y, \tau C)$, the action of $f$ on $\mu$ is given by $\tau(\underline{f}) \cdot \mu$. Then there exists finitely many $\eta_{1}, \eta_{2}, \ldots, \eta_{n}$ in $\operatorname{Ext}_{\mathcal{C}}^{1}(Y, \tau C)$ such that $\underline{H}^{\perp}=\sum_{i=1}^{n} \eta_{i} \Gamma_{C}$. Assume that $\eta_{i}$ is represented by the conflation $\tau C \rightarrow X_{i} \stackrel{\alpha_{i}}{\longrightarrow} Y$ for each $i=1,2, \ldots, n$. We have that $\alpha_{i}$ is right $\tau^{-} \tau C$-determined by Lemma 4.2. Then $\alpha_{i}$ is right $C$-determined by Proposition 3.3 . We observe that $\bigoplus_{i=1}^{n} \alpha_{i}$ is a deflation. By Lemma 2.1 we have that $\bigoplus_{i=1}^{n} \alpha_{i}$ is right $C$-determined.

Consider the following commutative diagram obtained by a pullback

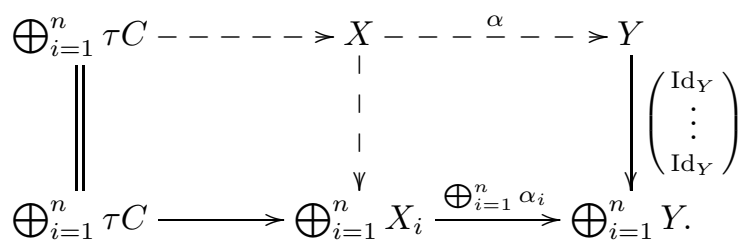

We have that $\alpha$ is a deflation and $\operatorname{Ker} \alpha$ lies in $\operatorname{add}(\tau C)$. By Lemma 2.2, the deflation $\alpha$ is right $C$-determined. By a direct verification, we have

$$
\operatorname{Im} \operatorname{Hom}_{\mathcal{C}}(C, \alpha)=\bigcap_{i=1}^{n} \operatorname{Im}_{\operatorname{Hom}}\left(C, \alpha_{i}\right) .
$$

For each $i=1,2, \ldots, n$, we set

$$
{ }^{\perp}\left(\eta_{i} \Gamma_{C}\right)=\left\{\underline{h} \in \underline{\operatorname{Hom}}_{\mathcal{C}}(C, Y) \mid \phi_{Y}(\underline{h})(\mu)=0 \text { for each } \mu \in \eta_{i} \Gamma_{C}\right\} .
$$

We observe that ${ }^{\perp}\left(\eta_{i} \Gamma_{C}\right)$ is a left $\Gamma_{C}$-submodule of $\underline{\operatorname{Hom}}_{\mathcal{C}}(C, Y)$. We mention that $\mathcal{P}(C, Y) \subseteq \operatorname{Im}_{\operatorname{Hom}}\left(C, \alpha_{i}\right)$, since $\alpha_{i}$ is a deflation. We claim that

$$
{ }^{\perp}\left(\eta_{i} \Gamma_{C}\right)=\operatorname{Im~Hom}_{\mathcal{C}}\left(C, \alpha_{i}\right) / \mathcal{P}(C, Y) .
$$

Let $h: C \rightarrow Y$ be a morphism in $\operatorname{Im}_{\operatorname{Hom}}\left(C, \alpha_{i}\right)$. We obtain that $\eta_{i} . h$ splits. Then we have

$$
\phi_{Y}(\underline{h})\left(\tau(\underline{f}) \cdot \eta_{i}\right)=\gamma\left(\left(\tau(\underline{f}) \cdot \eta_{i}\right) \cdot h\right)=\gamma\left(\tau(\underline{f}) \cdot\left(\eta_{i} \cdot h\right)\right)=0,
$$

for each $f: C \rightarrow C$. It follows that $\operatorname{Im}_{\operatorname{Hom}}\left(C, \alpha_{i}\right) / \mathcal{P}(C, Y) \subseteq{ }^{\perp}\left(\eta_{i} \Gamma_{C}\right)$.

On the other hand, let $h: C \rightarrow Y$ be a morphism such that $\underline{h} \in{ }^{\perp}\left(\eta_{i} \Gamma_{C}\right)$. Then we have $\phi_{Y}(\underline{h})\left(\tau(\underline{f}) \cdot \eta_{i}\right)=0$ for each $f: C \rightarrow C$. By the definition of $\tau$ in [6, Section 3], we have the following commutative diagram

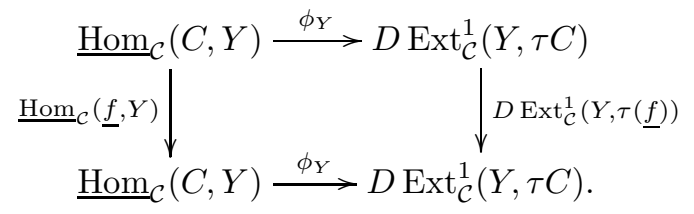

By a diagram chasing, we have

$$
\begin{aligned}
\phi_{Y}(\underline{h} \circ \underline{f}) & =\phi_{Y}\left(\underline{\operatorname{Hom}}_{\mathcal{C}}(\underline{f}, Y)(\underline{h})\right) \\
& =\left(D \operatorname{Ext}_{\mathcal{C}}^{1}(Y, \tau(\underline{f})) \circ \phi_{Y}\right)(\underline{h}) \\
& =\phi_{Y}(\underline{h}) \circ \operatorname{Ext}_{\mathcal{C}}^{1}(Y, \tau(\underline{f})) .
\end{aligned}
$$


Then for each $\eta_{i}$, we have

$$
\phi_{Y}(\underline{h} \circ \underline{f})\left(\eta_{i}\right)=\phi_{Y}(\underline{h})\left(\tau(\underline{f}) \cdot \eta_{i}\right)=0 .
$$

It follows that

$$
\phi_{C}(\underline{f})\left(\eta_{i} \cdot h\right)=\gamma\left(\left(\eta_{i} \cdot h\right) . f\right)=\gamma\left(\eta_{i} \cdot(h \circ f)\right)=\phi_{Y}(\underline{h \circ f})\left(\eta_{i}\right)=0 .
$$

We observe that $\phi_{C}(f)$ runs over all maps in $D \operatorname{Ext}_{\mathcal{C}}^{1}(C, \tau C)$, when $f$ runs over all morphisms in $\underline{\operatorname{End}}_{\mathcal{C}}(\bar{C})$. It follows that $\eta_{i} . h$ splits. In other words, the morphism $h$ factors through $\alpha_{i}$. We then obtain $h \in \operatorname{Im} \operatorname{Hom}_{\mathcal{C}}\left(C, \alpha_{i}\right)$. It follows that ${ }^{\perp}\left(\eta_{i} \Gamma_{C}\right) \subseteq$ $\operatorname{Im} \operatorname{Hom}_{\mathcal{C}}\left(C, \alpha_{i}\right) / \mathcal{P}(C, Y)$.

We observe that

$$
\underline{H}={ }^{\perp}\left(\underline{H}^{\perp}\right)={ }^{\perp}\left(\sum_{i=1}^{n} \eta_{i} \Gamma_{C}\right)=\bigcap_{i=1}^{n}\left(\eta_{i} \Gamma_{C}\right)
$$

where the first equality follows from the isomorphism $\phi_{Y}$. It follows that

$$
\underline{H}=\bigcap_{i=1}^{n} \operatorname{Im}_{\operatorname{Hom}}\left(C, \alpha_{i}\right) / \mathcal{P}(C, Y)=\operatorname{Im}_{\operatorname{Hom}_{\mathcal{C}}}(C, \alpha) / \mathcal{P}(C, Y) .
$$

Then the result follows since $\mathcal{P}(C, Y) \subseteq H$.

\section{A ChARACTERIZATION FOR DETERMined DEFlation}

Let $\mathcal{C}$ be a Hom-finite Krull-Schmidt exact category. We give a characterization for a deflation being right $C$-determined for some object $C$.

The following lemma, which is due to [2, Proposition XI.2.4 and Lemma XI.2.1], shows that each right $C$-determined morphism has a minimal right determiner.

Lemma 5.1. Let $f: X \rightarrow Y$ be a morphism, which is right $C$-determined for some object $C$.

(1) Assume that $C^{\prime}$ is an indecomposable object and $f^{\prime}: C^{\prime} \rightarrow Y$ almost factors through $f$. Then $C^{\prime}$ is a direct summand of $C$.

(2) Assume that $\left\{C_{1}, C_{2}, \ldots, C_{t}\right\}$ is a complete set of pairwise non-isomorphic indecomposable objects such that there exists some morphism $f_{i}: C_{i} \rightarrow Y$, which almost factors through $f$. Then $\bigoplus_{i=1}^{t} C_{i}$ is a minimal right determiner of $f$.

Recall that a morphism $f: X \rightarrow Y$ is called right minimal, if each $g \in \operatorname{End}_{\mathcal{C}}(X)$ with $f \circ g=f$ is an automorphism. Dually, we call $f$ left minimal, if each $g \in$ $\operatorname{End}_{\mathcal{C}}(Y)$ with $g \circ f=f$ is an automorphism.

Two morphisms $f: X \rightarrow Y$ and $f^{\prime}: X^{\prime} \rightarrow Y$ are called right equivalent if $f$ factors through $f^{\prime}$ and $f^{\prime}$ factors through $f$. Assume that $f$ and $f^{\prime}$ are right equivalent. Given an object $C$, we mention that $f$ is right $C$-determined if and only if so is $f^{\prime}$. We observe that $\mathcal{C}$ has split idempotents; see [8, Corollary 4.4]. By 4. Proposition 7.6], we have that $f$ is a deflation if and only if so is $f^{\prime}$.

Given a morphism $f: X \rightarrow Y$, we call a right minimal morphism $f^{\prime}: X^{\prime} \rightarrow Y$ the right minimal version of $f$, if $f$ and $f^{\prime}$ are right equivalent. Following [11, Section 2], we call Ker $f^{\prime}$ the intrinsic kernel of $f$.

We mention that each morphism $f: X \rightarrow Y$ has a right minimal version; see [3. Theorem 1]. Indeed, by the projectivization in [2, Section II.2], we have an equivalence $F: \operatorname{add}(X \oplus Y) \rightarrow \operatorname{proj} \Gamma_{X \oplus Y}$, where $\operatorname{proj} \Gamma_{X \oplus Y}$ is the category of 
finitely generated projective $\Gamma_{X \oplus Y}$-modules. By [2, Proposition I.2.1], the morphism $F(f)$ has a right minimal version $g$ in $\operatorname{proj} \Gamma_{X \oplus Y}$. Then $F^{-1}(g)$ yields the required morphism, where $F^{-1}$ is a quasi-inverse of $F$.

Dually, two morphisms $f: X \rightarrow Y$ and $f^{\prime}: X \rightarrow Y^{\prime}$ are left equivalent if $f$ factors through $f^{\prime}$ and $f^{\prime}$ factors through $f$. We mention that there exists some left minimal morphism $g: X \rightarrow Z$ such that $f$ and $g$ are left equivalent. We call $g$ the left minimal version of $f$, and call Cok $g$ the intrinsic cokernel of $f$.

Lemma 5.2. Let $\alpha: X \rightarrow Y$ be a deflation and $C$ be an indecomposable object. Assume that there exists some morphism $f: C \rightarrow Y$ which almost factors through $\alpha$. Then there exists an almost split conflation $K \rightarrow E \rightarrow C$ such that $K$ is a direct summand of the intrinsic kernel of $\alpha$.

Proof. We may assume that $\alpha$ is right minimal. Let $\beta: Z \rightarrow C$ be the pullback of $\alpha$ along $f$. Then $\beta$ is a deflation. By Proposition 2.3. we have that $\beta$ is right almost split. Let $\gamma: E \rightarrow C$ be the right minimal version of $\beta$. We observe that $\gamma$ is a right almost split deflation and $\operatorname{Ker} \gamma$ is a direct summand of $\operatorname{Ker} \beta \simeq \operatorname{Ker} \alpha$.

We claim that $\delta: \operatorname{Ker} \gamma \stackrel{\phi}{\rightarrow} E \stackrel{\gamma}{\rightarrow} C$ is an almost split conflation; see [1, Proposition II.4.4]. Indeed, let $f: \operatorname{Ker} \gamma \rightarrow K^{\prime}$ be a non-section. Assume that $f$ does not factor through $\phi$. Then the conflation $f . \delta$ does not split. In particular, the deflation $\gamma^{\prime}$ does not split, and hence factors through $\gamma$. We then obtain the following commutative diagram

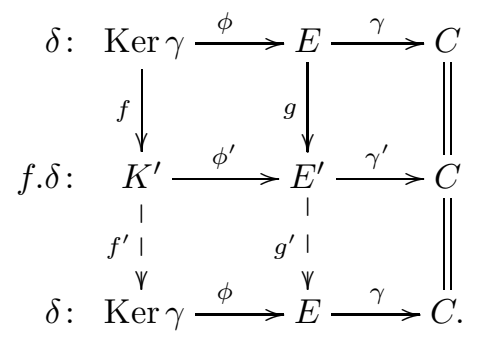

Since $\gamma$ is right minimal, the morphism $g^{\prime} \circ g$ is an isomorphism. We obtain that $f^{\prime} \circ f$ is an isomorphism, which is a contradiction since $f$ is not a section. It follows that $f$ factors through $\phi$. We obtain that $\phi$ is left almost split, and then $\delta$ is an almost split conflation.

Corollary 5.3. A minimal right determiner of a deflation has no nonzero projective direct summands and lies in $\mathcal{C}_{r}$.

Proof. Let $C$ be a minimal right determiner of a deflation $\alpha: X \rightarrow Y$. It is sufficient to show that each indecomposable direct summand $C^{\prime}$ of $C$ is non-projective and lies in $\mathcal{C}_{r}$. By Lemma 5.1, there exists some morphism $f: C^{\prime} \rightarrow Y$ which almost factors through $\alpha$. We observe that $C^{\prime}$ is not projective, since $f$ does not factor through $\alpha$. By Lemma [5.2, there exists an almost split conflation ending at $C^{\prime}$. Then the result follows from Lemma 4.1(1).

It is well known that in the category of finitely generated modules over an artin algebra, each morphism is right $C$-determined for some object $C$; see [2, Corollary XI.1.4] and [11, Theorem 1]. Here, we can only give a characterization for a deflation being right $C$-determined for some object $C$. 
Theorem 5.4. A deflation $\alpha: X \rightarrow Y$ is right $C$-determined for some object $C$ if and only if the intrinsic kernel of $\alpha$ lies in $\mathcal{C}_{l}$.

Proof. For the sufficiency, let $\alpha^{\prime}: X^{\prime} \rightarrow Y$ be the right minimal version of $\alpha$. We observe that $\alpha^{\prime}$ is still a deflation and $\operatorname{Ker} \alpha^{\prime} \in \mathcal{C}_{l}$. By Lemma 4.2. we have that $\alpha^{\prime}$ is right $\tau^{-}\left(\operatorname{Ker} \alpha^{\prime}\right)$-determined. It follows that $\alpha$ is right $\tau^{-}\left(\operatorname{Ker} \alpha^{\prime}\right)$-determined since $\alpha$ and $\alpha^{\prime}$ are right equivalent.

For the necessity, we may assume that $C$ is a minimal right determiner of $\alpha$. By Corollary 5.3. we have $C \in \mathcal{C}_{r}$. We observe that $\operatorname{Im}_{\operatorname{Hom}_{\mathcal{C}}}(C, \alpha)$ is a $\Gamma_{C}$-submodule of $\operatorname{Hom}_{\mathcal{C}}(C, Y)$. Since $\alpha$ is a deflation, we have $\mathcal{P}(C, Y) \subseteq \operatorname{Im}_{\operatorname{Hom}}(C, \alpha)$. By Theorem 4.3, there exists some deflation $\alpha^{\prime}: X^{\prime} \rightarrow Y$, which is right $C$-determined such that $\operatorname{Ker} \alpha^{\prime} \in \operatorname{add}(\tau C)$ and $\operatorname{Im} \operatorname{Hom}_{\mathcal{C}}(C, \alpha)=\operatorname{Im}_{\operatorname{Hom}}\left(C, \alpha^{\prime}\right)$. We observe that $\alpha \circ f$ factors through $\alpha^{\prime}$ and $\alpha^{\prime} \circ f^{\prime}$ factors through $\alpha$ for any $f: C \rightarrow X$ and $f^{\prime}: C \rightarrow X^{\prime}$. Since $\alpha$ and $\alpha^{\prime}$ are right $C$-determined, we have that $\alpha$ and $\alpha^{\prime}$ factor through each other. It follows that $\alpha$ and $\alpha^{\prime}$ are right equivalent. The intrinsic kernel of $\alpha$ is also the intrinsic kernel of $\alpha^{\prime}$, which is a direct summand of $\operatorname{Ker} \alpha^{\prime}$. Then the result follows since $\operatorname{Ker} \alpha^{\prime} \in \operatorname{add}(\tau C)$ and $\tau C \in \mathcal{C}_{l}$.

Example 5.5. Let $Q$ be the following infinite quiver

$$
\stackrel{\circ}{1} \longrightarrow \underset{2}{\circ} \longrightarrow \cdots \longrightarrow \underset{n}{\circ} \longrightarrow \cdots
$$

We consider the representations of $Q$ over a field. For each $i \geq 1$, we denote by $P_{i}$ the indecomposable projective representation and by $S_{i}$ the simple representation corresponding to $i$. Let $\mathcal{C}$ be the category of finitely presented representations. It is well known that $\mathcal{C}$ is a Hom-finite Krull-Schmidt abelian category.

For each $i \geq 1$, we consider the projective cover $f_{i}: P_{i} \rightarrow S_{i}$. We have $\operatorname{Ker} f_{i} \simeq$ $P_{i+1}$. By [6, Proposition 4.4], we have that $P_{i+1} \notin \mathcal{C}_{l}$. By Theorem [5.4, we have that $f_{i}$ is not right $C$-determined for any object $C$.

By Lemma 5.2, for any indecomposable object $C$, any morphism $g: C \rightarrow S_{i}$ does not almost factor through $f_{i}$. We observe that $f_{i}$ is not right 0 -determined. Then we have that the condition " $f$ is right $C$-determined for some object $C$ " for Lemma 5.1(2) is necessary.

\section{More Descriptions of OBJECTS In $\mathcal{C}_{r}$ And $\mathcal{C}_{l}$}

Let $\mathcal{C}$ be a Hom-finite Krull-Schmidt exact category. We will give some characterizations for an object lying in $\mathcal{C}_{r}$ or $\mathcal{C}_{l}$ via morphisms determined by objects.

Proposition 6.1. Let $K$ be an object without nonzero injective direct summands. Then $K$ lies in $\mathcal{C}_{l}$ if and only if there exists some deflation $\alpha: X \rightarrow Y$, which is right $C$-determined for some object $C$ such that $K$ is the intrinsic kernel of $\alpha$.

Proof. The sufficiency follows from Theorem 5.4. For the necessity, we assume $K \in \mathcal{C}_{l}$. Decompose $K$ as the direct sum of indecomposable objects $K_{1}, K_{2}, \ldots, K_{n}$. We have that each $K_{i}$ is non-injective. Then for each $i=1,2, \ldots, n$, there exists a non-split conflation $K_{i} \rightarrow X_{i} \stackrel{\alpha_{i}}{\longrightarrow} Y_{i}$. We observe that $\bigoplus_{i=1}^{n} \alpha_{i}$ is a deflation. By Lemma 4.2, we have that $\bigoplus_{i=1}^{n} \alpha_{i}$ is right $\tau^{-} K$-determined. We observe that $\alpha_{i}$ is right minimal. Then $\bigoplus_{i=1}^{n} \alpha_{i}$ is also right minimal. It follows that $K$ is the intrinsic kernel of $\bigoplus_{i=1}^{n} \alpha_{i}$.

The following lemma is the converse of Theorem 4.3 . 
Lemma 6.2. Let $C$ be an object. Assume that for each object $Y$ and each $\Gamma_{C^{-}}$ submodule $H$ of $\operatorname{Hom}_{\mathcal{C}}(C, Y)$ satisfying $\mathcal{P}(C, Y) \subseteq H$, there exists some deflation $\alpha: X \rightarrow Y$, which is right $C$-determined such that $H=\operatorname{Im}_{\mathcal{C}} \operatorname{Hom}_{\mathcal{C}}(C, \alpha)$. Then the object $C$ lies in $\mathcal{C}_{r}$.

Proof. It is sufficient to show that each non-projective indecomposable direct summand $C^{\prime}$ of $C$ lies in $\mathcal{C}_{r}$. Then each $f \in \mathcal{P}\left(C, C^{\prime}\right)$ is not a retraction. We observe that $\operatorname{rad}_{\mathcal{C}}\left(C, C^{\prime}\right)$ is formed by non-retractions. We obtain $\mathcal{P}\left(C, C^{\prime}\right) \subseteq \operatorname{rad}_{\mathcal{C}}\left(C, C^{\prime}\right)$.

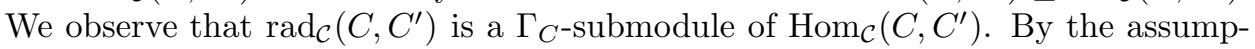
tion, there exists some deflation $\alpha: X \rightarrow C^{\prime}$, which is right $C$-determined such that $\operatorname{rad}_{\mathcal{C}}\left(C, C^{\prime}\right)=\operatorname{Im}_{\operatorname{Hom}_{\mathcal{C}}}(C, \alpha)$.

We claim that $\operatorname{Id}_{C^{\prime}}$ almost factors through $\alpha$. Indeed, the deflation $\alpha$ is not a retraction, since $\operatorname{Im} \operatorname{Hom}_{\mathcal{C}}(C, \alpha)=\operatorname{rad}_{\mathcal{C}}\left(C, C^{\prime}\right)$ is a proper submodule of $\operatorname{Hom}_{\mathcal{C}}\left(C, C^{\prime}\right)$. It follows that $\operatorname{Id}_{C^{\prime}}$ does not factor through $\alpha$. Let $f: T \rightarrow C^{\prime}$ be a morphism in $\operatorname{rad}_{\mathcal{C}}\left(T, C^{\prime}\right)$. For each $g: C \rightarrow T$, the morphism $f \circ g$ lies in $\operatorname{rad}_{\mathcal{C}}\left(C, C^{\prime}\right)=$ $\operatorname{Im~}_{\operatorname{Hom}}(C, \alpha)$. It follows that $f \circ g$ factors through $\alpha$. Since $\alpha$ is right $C$ determined, we have that $f$ factors through $\alpha$. It follows that $\operatorname{Id}_{C^{\prime}}$ almost factors through $\alpha$. By Lemma 5.2, there exists an almost split conflation ending at $C^{\prime}$. Then the result follows from Lemma 4.1(1).

Collecting the results obtained so far, we list some some characterizations for an object lying in $\mathcal{C}_{r}$. We mention that the equivalence between Theorem 6.3(5) and Theorem 6.3 (6) is somehow surprising.

Theorem 6.3. Let $\mathcal{C}$ be a Hom-finite Krull-Schmidt exact category and let $C \in \mathcal{C}$. The following statements are equivalent.

(1) The object $C$ lies in $\mathcal{C}_{r}$.

(2) For each object $Y$ and each $\Gamma_{C}$-submodule $H$ of $\operatorname{Hom}_{\mathcal{C}}(C, Y)$ satisfying $\mathcal{P}(C, Y) \subseteq H$, there exists some deflation $\alpha: X \rightarrow Y$, which is right $C$ determined such that $H=\operatorname{Im}_{\operatorname{Hom}}(C, \alpha)$.

(3) Each inflation $\alpha: X \rightarrow Y$ with $\operatorname{Cok} \alpha \in \operatorname{add} C$ is left $K$-determined for some object $K$.

If moreover $C$ is non-projective indecomposable, they are equivalent to the following statements.

(4) There exists an inflation $\alpha: X \rightarrow Y$ whose intrinsic cokernel is $C$ such that $\alpha$ is left $K$-determined for some object $K$.

(5) There exists an almost split conflation ending at $C$.

(6) There exists a non-split deflation which is right $C$-determined.

(7) There exists a deflation $\alpha: X \rightarrow Y$ and a morphism $f: C \rightarrow Y$ such that $f$ almost factors through $\alpha$.

Proof. By Theorem 4.3 and Lemma 6.2, we have "(1) $\Leftrightarrow(2)$ ".

By the dual of Lemma 4.2, we have "(10) $\Rightarrow(3)$ ". For each indecomposable nonprojective direct summand $C^{\prime}$ of $C$, there exists some non-split inflation $\alpha: X \rightarrow Y$ such that $\operatorname{Cok} \alpha \simeq C^{\prime}$. We observe that $\alpha$ is left minimal, since $\alpha$ is non-split and $C^{\prime}$ is indecomposable. By the dual of Theorem 5.4 we have that $C^{\prime} \in \mathcal{C}_{r}$. Then "(3) $\Rightarrow$ (11)" follows.

Now, we assume that $C$ is non-projective indecomposable. Then the dual of Proposition 6.1 implies "(1) $\Leftrightarrow$ (4)", and Lemma 4.1(1) implies "(1) $\Leftrightarrow$ (5)". 
It is well known that the right almost split deflation ending at $C$ is non-split and right $C$-determined; also see Lemma 4.2 and Proposition 3.3 . Then we have "(5) $\Rightarrow$ (6)". Let $\alpha$ be a non-split right $C$-determined deflation. We observe by Lemma 5.1 that $C$ is a minimal right determiner of $\alpha$. By Lemma 5.1, we have "(6) $\Rightarrow(7)$ ". By Lemma [5.2, we have "(17) $\Rightarrow$ (5)".

\section{Exact Categories having Auslander-Reiten duality}

Let $\mathcal{C}$ be a Hom-finite Krull-Schmidt exact category. Following [9, Definition 2.6] and [5, Definition 3.1], we introduce the following notion.

Definition 7.1. An exact category $\mathcal{C}$ is said to have right stably determined deflations if for each object $Y$ the following conditions hold.

(1) Each deflation ending at $Y$ is right $C$-determined for some object $C$.

(2) For each object $C$ and each $\Gamma_{C}$-submodule $H$ of $\operatorname{Hom}_{\mathcal{C}}(C, Y)$ such that $\mathcal{P}(C, Y) \subseteq H$, there exists some deflation $\alpha: X \rightarrow Y$, which is right $C$ determined such that $H=\operatorname{Im}_{\operatorname{Hom}_{\mathcal{C}}}(C, \alpha)$.

Dually, an exact category $\mathcal{C}$ is said to have left stably determined inflations if the opposite category $\mathcal{C}^{\mathrm{op}}$ has right stably determined deflations.

We give the following characterizations for an exact category having AuslanderReiten duality in the sense of [10].

Theorem 7.2. Let $\mathcal{C}$ be a Hom-finite Krull-Schmidt exact category. The following statements are equivalent.

(1) $\mathcal{C}$ has Auslander-Reiten duality.

(2) $\mathcal{C}$ has right stably determined deflations.

(3) $\mathcal{C}$ has left stably determined inflations.

Proof. We observe that $\mathcal{C}$ has Auslander-Reiten duality in the sense of 10 if and only if $\mathcal{C}_{l}=\mathcal{C}=\mathcal{C}_{r}$. The dual of the equivalence between Theorem 6.3 (1) and Theorem 6.3(3) implies that an object $K$ lies in $\mathcal{C}_{l}$ if and only if each deflation whose kernel lies in add $K$ is right $C$-determined for some $C$. Combining this with the equivalence between Theorem 6.3(1) and Theorem 6.3(2), we have "(11) $\Leftrightarrow$ (2)".

By duality, we have "(10) $\Leftrightarrow(3)$ ".

Remark 7.3. Compared with [5, Theorem 3.4], it is somewhat surprising that Theorem 7.2(2) and Theorem 7.2(3) are equivalent. It seems that Definition 7.1(1) and Definition 7.1(2) are more symmetric than the conditions (REC1) and (REC2) in [5, Definition 3.1].

\section{ACKNOWLEDGEMENTS}

We are very grateful to the referee for many helpful suggestions. The first author thanks his advisor Professor Xiao-Wu Chen for his guidance and encouragement. We thank Dawei Shen for helpful comments. The work is supported by National Natural Science Foundation of China (Nos. 11571329 and 11671174).

\section{REFERENCES}

[1] M. Auslander, Functors and morphisms determined by objects, in Representation theory of algebras (Proc. Conf., Temple Univ., Philadelphia, Pa., 1976), vol. 37 of Lecture Notes in Pure Appl. Math., Dekker, New York, 1978, 1-244. 
[2] M. Auslander, I. Reiten, and S. O. Smalø, Representation theory of Artin algebras, vol. 36 of Cambridge Studies in Advanced Mathematics, Cambridge Univ. Press, Cambridge, 1995.

[3] N. Bian, Right minimal morphisms in arbitrary Krull-Schmidt categories, J. Math. (Wuhan) 29 (2009), 577-580.

[4] T. Bühler, Exact categories, Expo. Math. 28 (2010), 1-69.

[5] X.-W. Chen and J. Le, A note on morphisms determined by objects, J. Algebra 428 (2015), $138-148$

[6] P. Jiao, The generalized Auslander-Reiten duality on an exact category, preprint (2016), arXiv:1609.07732

[7] B. Keller, Chain complexes and stable categories, Manuscripta Math. 67 (1990), 379-417.

[8] H. Krause, Krull-Schmidt categories and projective covers, Expo. Math. 33 (2015), 535-549.

[9] H. Krause, Morphisms determined by objects in triangulated categories, in Algebras, quivers and representations, vol. 8 of Abel Symp., Springer, Heidelberg, 2013, 195-207.

[10] H. Lenzing and R. Zuazua, Auslander-Reiten duality for abelian categories, Bol. Soc. Mat. Mexicana (3) 10 (2004), 169-177.

[11] C. M. Ringel, Morphisms determined by objects: the case of modules over Artin algebras, Illinois J. Math. 56 (2012), 981-1000.

[12] C. M. Ringel, The Auslander bijections: how morphisms are determined by modules, Bull. Math. Sci. 3 (2013), 409-484.

School of Mathematical Sciences, University of Science and Technology of China, HeFei 230026, PR ChinA

E-mail address: jiaopjie@mail.ustc.edu.cn

School of Mathematical Sciences, University of Science and Technology of China, HeFei 230026, PR China

E-mail address: juele@ustc.edu.cn 